\title{
Aspectos determinantes no consumo
}

\section{masstige}

\section{Solon Bevilacqua}

\section{RESUMO}

Esse estudo investigou o consumo masstige sob a lente do egoísmo ético, intimamente relacionado ao prazer e associado aos construtos diferenciação, exclusividade, funcional (atributos), status e tradição. Efetuou-se uma validação inicial a partir do emprego de um instrumento padronizado, estruturado pelas cinco dimensões relacionadas. O quadro teórico contemplou principalmente autores evolucionistas, cuja discussão centrou-se em torno da natureza e cultura, para uma dimensão de consumo intitulada prazer - egoísmo ético. A análise de dados foi realizada a partir do emprego de Modelagem de Equações Estruturais com estimação PLS. Os achados da pesquisa indicam que a dimensão prazer - egoísmo ético para o consumo masstige é estruturada pela tradição; status e características funcionais do produto, enquanto a exclusividade não se apresenta significativa para o universo dos bens e serviços de prestígio e acessíveis.

Palavras-chave: prazer; luxo; consumo; Masstige; MEE PLS

Decisive aspects in the masstige consumption

\section{ABSTRACT}

This study investigated the mass consumption under the lens of ethical egoism, closely related to pleasure and associated with differentiation, exclusivity, functional (attributes), status, and tradition constructs. The theoretical framework contemplated mainly evolutionary authors, focusing the discussion on nature and culture, to a dimension of consumption titled pleasure - ethical egoism. Data analysis was performed using Structural Equation Modeling with PLS estimation. The research findings indicate that the dimension pleasure - ethical egoism for masstige consumption - is structured by tradition; status and functional characteristics of the product, whereas exclusivity is not significant for the universe of prestige and affordable goods and services.

Keywords: pleasure; lux; consumption; masstige; MEE PLS

Recebido em: 27/11/2018 Revisado em: 29/04/2019 Aprovado em: 14/04/2020

Check for updates
Solon Bevilacqua (iD),

Universidade Federal de Goiás, Brasil Doutorado em Psicologia pela Pontifícia Universidade Católica de Goiás, Brasil.

solbev@gmail.com 


\section{Introdução}

As pesquisas recentes relacionadas aos produtos de prestígio, mas acessíveis (masstige), indicam que esse consumo ocorre em função de uma busca por qualidade superior e prazer auto direcionado. Esse prazer é construído ao apresentar-se quanto foi pago pelo produto, quando ocorre imitação de admirados consumidores ou pelo espírito de competição compartilhada (Amatulli \& Guido, 2011). Forças internas impulsionam a motivação para a autoestima, que apenas o prazer auto direcionado pode satisfazer (Silverstein \& Fiske, 2003). Entender os aspectos que envolvem esse mercado possibilita desenvolver novos produtos, melhorar a qualidade dos processos e, fundamentalmente, abrir novos mercados.

Nesta pesquisa centrada no consumo masstige, seis construtos são analisados: prazer, diferenciação, exclusividade, funcional, status e tradição. Objetiva-se com esta pesquisa investigar as motivações dos consumidores na dimensão prazer-autoestima no consumo masstige.

A presente pesquisareduzaatual lacuna de conhecimento, desenvolvendo e validando um modelo empírico que testa os efeitos de certas motivações no consumo masstige. A questão da tradição das grandes marcas, atualmente relegada ao segundo plano, em virtude da aceleração no lançamento de novos produtos, emerge de os dados como aspecto preponderante para o constructo prazer-autoestima. As hipóteses testadas relacionam-se ao prazer, exclusividade, diferenciação; funcional (preço, qualidade, garantia...) e tradição.

A abordagem utilizada nesta pesquisa buscou esclarecer as seis dimensões relacionadas ao consumo masstige. Entende-se que consumir é ir além de comprar e satisfazer necessidades e desejos (Solomon, Russel-Bennett, \& Previte, 2002); que gestores de marketing transformaram-se em gerentes de significados (McCracken, 1986); que o homem é um ser biologicamente cultural (Pinker, 2009), além de economicamente social (Douglas \& Isherwood, 2002). A partir desse pressuposto, o dinamismo que envolve o estudo do comportamento humano deveria acomodar aspectos do individualismo, tais como a busca pelo prazer, mas também questões como o consumo simbólico.

No decorrer deste artigo serão abordadas questões teóricas que suportam as 4 hipóteses centradas na dimensão prazer. A metodologia descreve os procedimentos utilizados na pesquisa, realizada no Brasil Central, junto a 357 participantes e consumidores de produtos e serviços masstige; os dados são analisados com o emprego de Modelagem de Equações Estruturais (PLS); as considerações finais são apresentadas à luz da teoria do consumo motivado pelo prazer.

\section{Consumo Masstige e o construto Prazer}

Sem maiores variações conceituais, as pessoas compram produtos de luxo e masstige pelo mesmo motivo. As motivações externas (riqueza de sinal) dizem respeito aos casos em que o objetivo é essencialmente exibir 
publicamente o luxo, de modo a afirmar o status do consumidor, enquanto as motivações internas (auto recompensa) se relacionam com consumo de luxo baseado em emoções, estado de espírito e sensações subjetivas relacionadas à autopercepção de um indivíduo (Amatulli \& Guido, 2011).

Os produtos masstige, apesar de acessíveis, ainda desfrutam de um nível razoável de prestígio percebido, mas, ao mesmo tempo, são vendidos a preços que estão apenas ligeiramente acima dos produtos de massa. Uma estratégia de posicionamento masstige pode ser muito inovadora e eficaz, pois combina um posicionamento de prestígio com um apelo amplo, porém com pouca ou nenhuma diluição da marca. A questão crucial para o sucesso desta estratégia é manter o equilíbrio adequado entre a percepção de prestígio e preço premium (Truong, McColl, \& Kitchen, 2009).

O prazer em consumir funciona como uma alavanca no consumo de bens e serviços de prestígio (Smith, 1998; Mandeville, 2017). Esse prazer deve ser entendido como um sentimento de realização, de sentir-se único, de diferenciação, de status, além de firmar um sentimento de pertencimento. Esse conjunto de sentimentos consiste em um prazer egoísta ético, um sentimento que está imbricado na estrutura do gene humano; afinal, o homem é estruturado a partir de comportamento biológico e cultural (Pinker, 2009). Tal sentimento tem origem no acúmulo de coisas já pelos primeiros hominídeos (James, Burkhardt, Bowers, \& Skrupskelis, 2017). Sendo esse o alimento das classes que projetavam grupos seletos, os quais buscam distinção.Tais grupos prosperaram no contexto de sua cultura de consumo na busca do prazer e do status (Veblen, 2017).

O prazer relaciona-se com status, tradição, exclusividade, diferenciação e aspectos visuais que evidenciem qualidade, design inovador e raridade (Bourdieu, 1989). Ademais, o consumo simbólico constitui-se na transmissão dos exatos significados (McCracken, 1986). É perceptível que a visão de consumo vebleniano, centrado na ostentação e busca exacerbada pelo status, contemple apenas parte da questão.

A ideia do consumo conspícuo não previa ambientes simbólicos e culturais. Os consumidores eram vistos como indivíduos individualistas que almejavam a projeção a partir da exibição. Tratava-se de uma época de barbárie, mas quando a seguir o homem avançou para o status de bem-sucedido, agressivo, forte, atilado e intrépido, este conseguiu evitar o embrutecimento, cultivou o gosto e assim obteve destaque junto aos ignóbeis. A evolução egoísta empreendida proporcionou um senso estético sobre a forma correta de consumir, por conseguinte, apontou para uma vida de ócio corretamente conduzida (Veblen, 2017). A exibição é apenas uma das faces desse consumo específico.

Aquele que faz bom uso dos costumes corretos, deve preocupar-se com a emissão dos sinais adequados, de forma a promover a correta distinção pelos significados contidos nos bens (Bourdieu, 1989; McCracken, 1986). O prazer e a autoestima passaram a ser para o marketing uma verdadeira pedra angular.

O efeito Veblen é atual e ainda influencia as pesquisas sobre consumo, afinal, as pessoas que buscam status na compra de produtos posicionais 
também aspiram pertencer a novos grupos de referência. Alguns consumidores, em vez disso, buscam benefícios discretos, como a qualidade do produto (Beverland, 2006) e o prazer (Fiske \& Silverstein, 2004).

Status está relacionado ao fato de que quanto mais um ato de consumo é incomum, pouco renovado, inacessível, mais demonstra uma pulsão ou signo de consideração e distinção social. Tal grandeza de distinção sintetiza o pré-requisito para a identificação ou distinção com a classe social e imita-se a classe superior (planos de carreira ambiciosos) ou inferiores (regressão de comportamento) (Dubois, Czellar, \& Laurent, 2005).

A tradição é um item controvertido, mas presente no consumo por meio de seus fundadores e artesões, que transmitem seu legado para as demais gerações. Assim ocorreu com as marcas clássicas Hérmès (1837), Louis Vuitton (1896), Rolex (1905), Chanel (1909), Prada (1913), Gucci (1921), Dior (1946), Armani (1975), Versace (1978), entre outras (Serraf, 1991).

A partir do exposto, são relacionadas as duas primeiras hipóteses da pesquisa:

Hipótese 1: A tradição influencia o prazer no consumo posicional e masstige.

Hipótese 2: O status influencia o prazer no consumo posicional e masstige.

As duas hipóteses possuem estreita relação com o constructo prazer, variável dependente na pesquisa.

Se todos os homens são iguais diante da necessidade e diante do princípio da satisfação, essa igualdade só se exprime diante do valor de uso dos bens e dos serviços. Torna-se claro também que a busca pela exclusividade passa pela raridade ou pela estratégia de restringir a oferta. Às vezes a oferta é restrita pelo uso da matéria prima, mas muitas vezes é premeditada (Allérès, 2000). Tal exclusividade insere-se na dimensão prazer quando perfaz uma condição: a unidade de consumo. Este conceito prevê que acessórios sejam utilizados em conjunto, aliados a outros símbolos de distinção (tais como relógios, joias, carteira, relógio, gravatas, óculos, pulseiras, ternos, sapato...) (McCracken, 1986). A unidade completa o conceito de luxo, aliado ao uso que é dado ao bem. Aquele produto que é posto em uso diário, invariavelmente abandona o caráter de exclusividade, pois torna-se corriqueiro (Douglas \& Isherwood, 2006). A busca pela diferenciação é determinada pelo estilo e bom gosto. O estilo e o bom gosto determinam a diferenciação, a inclusão e a exclusão entre semelhantes (Bourdieu, 2005).

\section{Hipótese 3: A exclusividade influencia o prazer no consumo masstige.}

O aspecto funcional, fundamentalmente é definido por qualidade extrema. A particularidade do produto mais importante é sem dúvida a qualidade de sua estrutura e matéria prima, serviços de suporte e pós venda, podendo ser estendida ao preço, uma vez que promoções criam ou prejudicam o conceito masstige (Paul, 2019). Espera-se que o produto realmente seja durável e não apresente aparência de algo que apresente defeitos (Allérès, 2000). 
Hipótese 4: A diferenciação via aspectos funcionais do produto influencia o prazer no consumo posicional e masstige (entende-se por funcional as características físicas do produto, tais como design, qualidade, dimensões, peso, matéria prima etc.).

O sentimento de exclusividade, a busca pelo status e a diferenciação são aspectos formadores de uma dimensão intitulada prazer. São elementos construídos em torno de símbolos emitidos por elementos tangíveis e visuais (qualidade, preço, design etc.).

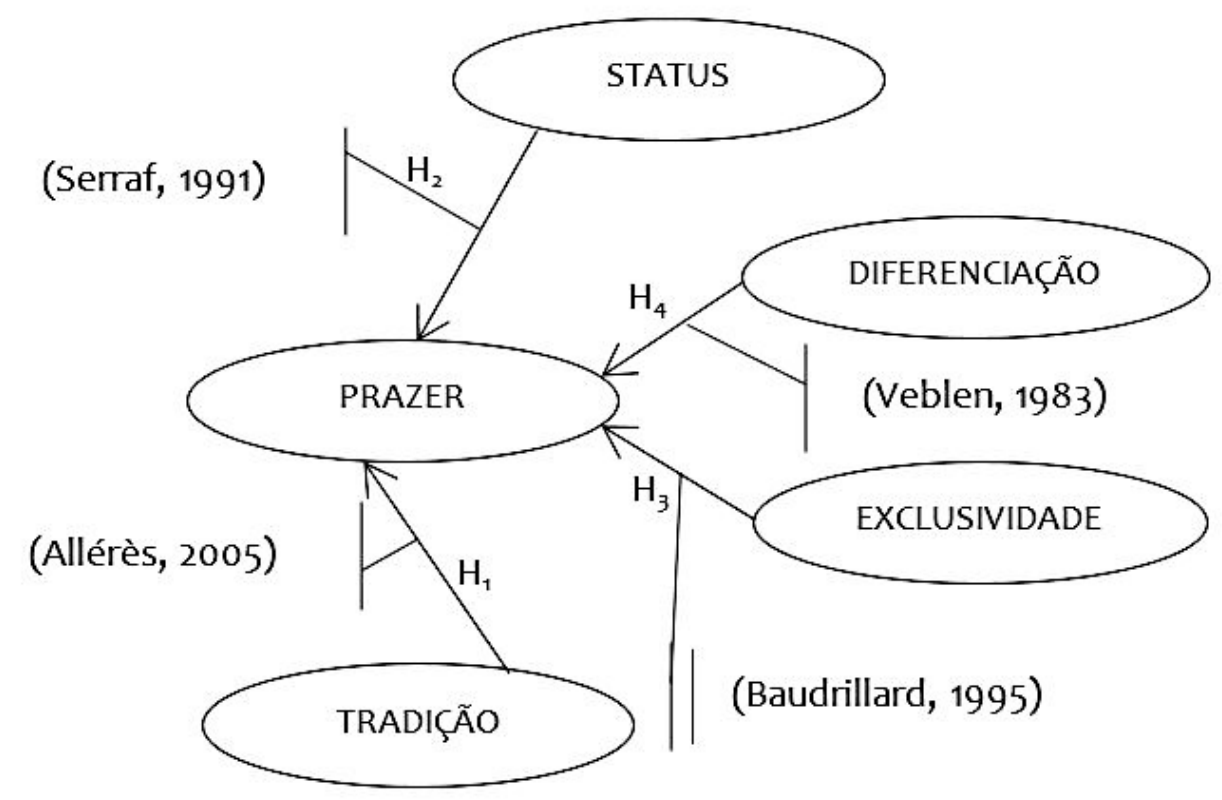

Figura 1. Hipóteses e a dimensão prazer-autoestima

Cabe destacar no modelo de hipóteses proposto que há uma confluência de variáveis não facilmente observáveis, orientadas para a busca do prazer. A abordagem teórica que se adapta melhor ao modelo seria aquela que prevê o consumidor um homo consumericus. Previsto dessa forma, seu comportamento é voraz, móvel, flexível, liberto das antigas culturas de classe, imprevisível nos seus gostos e nas suas compras e sedento de marcas, de autenticidade, de efemeridade, de comunicação de experiências emocionais e bem-estar (Lipovetsky \& Roux, 2005). Além disso, quando em perseguição de seu próprio interesse, alimenta boas práticas para a promoção do bem geral. Essa é a questão chave que norteia o egoísmo ético, argumento tornado famoso por Adam Smith e Bernard de Mandeville e ponto central para economistas como Milton Friedman e George Stigler (Giannetti, 1994).

Em virtude da reunião de variáveis independentes e dependentes de complexa definição, não diretamente observáveis, o conceito de construto apresenta-se como o mais adequado (uma vez que reúne em torno de si uma série de variáveis com o objetivo de clarificar um conceito). A opção metodológica proposta é a modelagem de equações estruturais. 
Procedimentos Metodológicos

Um modelo teórico foi criado para investigar a influência de fatores comportamentais no consumo de produtos e serviço masstige. A estrutura teórica foi desenvolvida com seis variáveis não diretamente observáveis, que representam construtos orientados ao prazer, variável endógena na estrutura. Cada relação do modelo foi testada a partir da técnica de Modelagem de Equações Estruturais, estimação PLS (Partial Least Squares-Path Modeling).

\section{Procedimentos específicos}

Investigou-se, de forma aleatória, uma amostra de 357 participantes (indivíduos maiores de 18 anos e consumidores frequentes de produtos masstige), na região de Goiânia, Goiás. Foi aplicado um questionário impresso com 35 questões, as quais foram desenvolvidas especificamente para esse projeto, utilizando a escala de 7 pontos. A coleta de dados ocorreu em eventos organizados com a temática centrada em consumo masstige. As palestras realizadas abordaram a temática desta pesquisa, evocando negócios e produtos posicionais, mas com preços acessíveis.

O instrumento estruturado foi elaborado com questões advindas de literatura especializada, centrada no consumo posicional e de distinção, a partir de autores como Allérès (2005); Dubois, Czellar e Laurent (2005); Baudrillard (1995); Lipovetsky e Roux (2005); Veblen (1983); Bourdieu (2005); Serraf (1991); e Douglas e Isherwood (2006).

\section{Preparação dos construtos e manipulação das variáveis}

Além das 35 questões em escala tipo Likert, (com variação concordo totalmente a discordo totalmente), questões demográficas foram inseridas ao término do questionário (sexo, idade e renda). Os pontos e sua origem estão no Quadro 1: 
Quadro 1: Estrutura do Instrumento

\begin{tabular}{|c|c|c|}
\hline Atributo & Autores & Dimensão \\
\hline 01. Presença de alta qualidade & (Lipovetsky \& Roux, 2005) & \multirow{13}{*}{$\begin{array}{l}\text { Atributos } \\
\text { (FUN) }\end{array}$} \\
\hline 02. Relação com alta tecnologia & (Allérès, 2005) & \\
\hline 03. Ter sido fabricado com matéria prima rara & (Lipovetsky \& Roux, 2005) & \\
\hline 04. Deve ser considerado uma arte & (Douglas \& Isherwood, 2006) & \\
\hline 05. Que reflita quem é seu dono & (Dubois, Czellar, \& Laurent, 2005 & \\
\hline 06. Serve à promoção da imagem pessoal & (Dubois, Czellar, \& Laurent, 2005) & \\
\hline 07. Representar uma tradição histórica & (Douglas \& Isherwood, 2006) & \\
\hline 08. Relação estreita com uma marca forte & (Serraf, 1991) & \\
\hline 09. Demonstrar o quanto a pessoa é bem-sucedida & (Veblen, 1983) & \\
\hline 10. Expressar quem o consumidor deseja ser & (Dubois, Czellar, \& Laurent, 2005) & \\
\hline 11. Ter um preço elevado & (Baudrillard, 1995) & \\
\hline 12. Ser raro & (Serraf, 1991) & \\
\hline 13. Ser de elevada sofisticação & (Lipovetsky \& Roux, 2005) & \\
\hline 14. Determinar quem tem dinheiro & (Dubois, Czellar, \& Laurent, 2005) & \multirow{7}{*}{$\begin{array}{l}\text { Difere } \\
\text { (DIF) }\end{array}$} \\
\hline 15. Ser consumido em momentos raros da vida & (Veblen, 1983) & \\
\hline 16. Criar diferenciação entre as pessoas & (Bourdieu, 1984) & \\
\hline 17. Possibilitar influenciar pessoas & (Bourdieu, 1984) & \\
\hline 18. Refletir um estilo de vida & (Bourdieu, 1984) & \\
\hline 19. Uma réplica [...] também é atraente & (Bourdieu, 1984) & \\
\hline 20. Ser usado para a competição entre as pessoas & (Dubois, Czellar, \& Laurent, 2005) & \\
\hline 21. Demanda conhecimento prévio de sua história & (Serraf, 1991) & \multirow{4}{*}{$\begin{array}{l}\text { Tradição } \\
\text { (TRA) }\end{array}$} \\
\hline 22. Eu sei muito sobre [...] as grandes marcas & (Serraf, 1991) & \\
\hline 23. Eu poderia falar sobre produtos especiais & (Dubois, Czellar, \& Laurent, 2005) & \\
\hline 24. Deve ser consumido de acordo com ambiente & (Castarède, 2005) & \\
\hline 25. Deve atrair admiração para quem o consome & (McClelland, 1978) & \multirow{4}{*}{$\begin{array}{l}\text { Prazer } \\
\text { (PRA) }\end{array}$} \\
\hline 26. Estar associado à emoção da vida & (McClelland, 1978) & \\
\hline 27. É necessário estar relacionado à boa reputação & (Douglas \& Isherwood, 2006) & \\
\hline 28. Envolve um verdadeiro prazer & (Douglas \& Isherwood, 2006) & \\
\hline 29. Deve revelar o bom gosto do consumidor & (McClelland, 1978) & \multirow{7}{*}{$\begin{array}{l}\text { Exclusivo } \\
\text { (EXC) }\end{array}$} \\
\hline 30. Expressa exclusividade & (McClelland, 1978) & \\
\hline 31. Uma posse para poucas pessoas & (Lipovetsky \& Roux, 2005) & \\
\hline 32. Com ele é possível fazer parte de um grupo especial & (Allérès, 2005) & \\
\hline 33. Deve estar ligado à ostentação & (Lipovetsky \& Roux, 2005) & \\
\hline 34. Sua posse acarreta poder & (Douglas \& Isherwood, 2006) & \\
\hline 35. Consiste numa realização pessoal & (Dubois, Czellar, \& Laurent, 2005) & \\
\hline
\end{tabular}

Tal levantamento possibilitou a ligação entre as variáveis latentes no diagrama de caminhos da Modelagem de Equações Estruturais (MEE). Após a primeira operação de Análise Fatorial, as dimensões previstas no Quadro 1 sofreram alterações (ver Tabela 2). 
O entendimento para a próxima etapa é que 5 dimensões sintetizam a operacionalização da pesquisa, que prevê as dimensões 'funcional', 'tradição', 'exclusividade', 'status' e 'diferenciação' para a construção do 'prazer'.

A análise de missing values e outliers provocou a exclusão de 12 casos. A opção escolhida se justifica pela adequada relação caso-variável de 10,2 (mínimo aceitável de 5 casos por variável), para Análise Fatorial, bem como pela questão de praticidade em não se derivar para processos de atribuição e análises decorrentes (Hair, Black, Babin, Anderson, \& Tatham, 1998). A avaliação da normalidade estatística através dos histogramas, box plots e teste de Kolmogorov-Smirnov, considerando $\mathrm{H}_{0}$ (os dados da variável originam-se de uma distribuição normal) e $\mathrm{H}_{1}$ (os dados da variável não se originam de uma distribuição normal), concluiu que os dados estavam em sua distribuição normal. Com a exclusão de outliers, a análise visual apresentouse favorável, assim como os valores do teste e Kolmogorov-Smirnov com correção de significância de Lilliefors.

Construída a matriz de correlação, o primeiro exame visual constatou que não há muitas correlações acima dos escores sugeridos, embora alguns sejam elevados como 0,50 e 0,60. Valores próximos de 0,50 indicam que os fatores encontrados não conseguem descrever satisfatoriamente as variações dos dados originais. A opção desejada para a extração de fatores recaiu sobre Ortogonal e Varimax. Nesse sentido, pelo critério da raiz latente, a extração seria de 6 fatores; pelo critério de Kaiser, a escolha seria 10. Os demais critérios sugeridos, de percentagem de variância e heterogeneidade dos respondentes, foram desconsiderados em virtude da baixa variância acumulada registrada na primeira tentativa de extração de fatores (56,75\%). Ao se rodar a Análise Fatorial sem determinação da quantidade de fatores a extrair, o máximo de variância total acumulada obtida foi de 56,75\%.

Tabela 1: Indicadores de Consistência

\begin{tabular}{ccccc}
\hline & R Square & Variância & KM0 & $\alpha$ \\
\hline Diferenciação & 0,212 & 15,90 & 0,874 & 0,668 \\
Exclusividade & 0,255 & 14,92 & 0,751 & 0,584 \\
Funcional & 0,301 & 5,97 & 0,717 & 0,637 \\
Prazer & 0,206 & 33,10 & 0,626 & 0,564 \\
Status & 0,000 & 14,82 & 0,651 & 0,649 \\
Tradição & 0,337 & 20,64 & 0,874 & 0,776 \\
\hline
\end{tabular}

Com relação ao $\alpha$, destaca-se 'tradição', 'diferenciação' e 'status' como valores expressivos, conferindo a ressalva que essa medida é extremamente sensível à medida que novas variáveis são inseridas nas dimensões de estudo. Entretanto, para o $R^{2}$ tem-se que status tem coeficiente de explicação em zero, ou seja, nada elucidativo.

A AVE melhorou significativamente. À medida que os procedimentos de exclusão para as variáveis foram ocorrendo, os construtos registraram melhora com o procedimento. As variáveis possuem cargas altamente significativas do ponto de vista estatístico ( $\mathrm{t}>1,96)$, conforme a Tabela 2 , parâmetro ( $t>1,96$ ou $p<0,05)$. 
Tabela 2: Estatística geral por construto

\begin{tabular}{ccccc}
\hline Construto & Variável & Carga Fatorial & $\varepsilon$ & t stat \\
\hline Diferenciação & DIF01 & 0,693 & 0,044 & 15,7 \\
Diferenciação & DIF02 & 0,687 & 0,050 & 13,7 \\
Diferenciação & DIF04 & 0,605 & 0,058 & 10,5 \\
Diferenciação & DIF07 & 0,658 & 0,048 & 13,8 \\
\hline Exclusividade & EXC01 & 0,647 & 0,048 & 13,6 \\
Exclusividade & EXC02 & 0,602 & 0,054 & 11,1 \\
Exclusividade & EXC03 & 0,655 & 0,048 & 13,8 \\
Exclusividade & EXC04 & 0,678 & 16,4 \\
\hline Funcional & FUN02 & 0,662 & 0,041 & 14,7 \\
Funcional & FUN03 & 0,806 & 0,045 & 34,5 \\
Funcional & FUN04 & 0,587 & 0,023 & 10,1 \\
Funcional & FUN05 & 0,716 & 0,058 & 17,6 \\
Funcional & PRA01 & 0,729 & 15,0 \\
\hline Prazer & PRA02 & 0,720 & 14,9 \\
Prazer & PRA03 & 0,743 & 0,049 & 17,1 \\
\hline Status & STA01 & 0,663 & 0,044 & 13,0 \\
Status & STA02 & 0,652 & 0,051 & 10,2 \\
Status & STA04 & 0,758 & 0,064 & 16,8 \\
Status & STA05 & 0,635 & 0,045 & 18,0 \\
\hline Tradição & TRA10 & 0,636 & 22,0 \\
Tradição & TRA02 & 0,712 & 0,069 & 15,7 \\
Tradiçãa & TRA03 & 0,610 & 0,032 & 23,4 \\
Tradiçãa & TRA04 & 0,720 & 0,039 & 20,8 \\
Tradição & TRA06 & 0,031 & 29,4 \\
Tradição & TRA07 & 0,033 & \\
Tradição & TRA08 & 0,677 & 0,035 & \\
\hline Para vien & 0,777 & & \\
\hline
\end{tabular}

Para verificar a validade discriminante no nível das variáveis criou-se uma diagonal com a raiz quadrada das AVE's, na matriz de correlação das dimensões. Como os valores na diagonal são maiores do que os valores fora da diagonal, há validade discriminante.

Tabela 3: Correlação entre Variáveis Latentes (Diagonal com a Raiz Quadrada das AVE)

\begin{tabular}{lcccccc}
\hline \multicolumn{1}{c}{ Dimensão } & Diferenciação & Exclusividade & Funcional & Prazer & Status & Tradição \\
\hline Diferenciação & $\mathbf{0 , 6 6 1}$ & & & & & \\
Exclusividade & 0,463 & $\mathbf{0 , 6 4 6}$ & & & & \\
Funcional & 0,293 & 0,379 & $\mathbf{0 , 6 9 7}$ & & & \\
Prazer & 0,270 & 0,362 & 0,334 & $\mathbf{0 , 7 3 1}$ & & \\
Status & 0,495 & 0,351 & 0,197 & 0,263 & $\mathbf{0 , 6 7 9}$ & \\
Tradição & 0,253 & 0,487 & 0,516 & 0,285 & $-0,005$ & $\mathbf{0 , 6 9 5}$ \\
AVE & 0,437 & 0,417 & 0,486 & 0,534 & 0,461 & 0,483 \\
\hline
\end{tabular}


A seguir fez-se necessário também avaliar a validade discriminante no nível dos indicadores. De acordo com a Tabela 4 são fatores preponderantes para a exclusividade questões relacionadas à diferenciação, assim como questões funcionais do produto estão relacionadas à exclusividade. Tradição é construída pela exclusividade e funcionalidade do produto.

Tabela 4: Correlação entre Variáveis Latentes

\begin{tabular}{ccccccc}
\hline & Diferenciação & Exclusividade & Funcional & Prazer & Status & Tradição \\
\hline Diferenciação & 1 & & & & & \\
Exclusividade & 0,459 & 1 & & & & \\
Funcional & 0,311 & 0,383 & 1 & & & \\
Prazer & 0,269 & 0,336 & 0,320 & 1 & & \\
Status & 0,460 & 0,399 & 0,272 & 0,293 & 1 & 1 \\
Tradição & 0,407 & 0,546 & 0,516 & 0,372 & 0,197 & 1 \\
\hline
\end{tabular}

Na elaboração dos construtos, a meta foi seguir o referencial teórico, utilizando a proposta da Análise Fatorial como suporte. O principal critério de avaliação da confiabilidade e da validação do modelo estrutural é por meio do coeficiente de determinação $R^{2}$ para as variáveis latentes endógenas (Chin, 1998; Hair et al., 1998)the IS field has seen a substantial increase in the number of submissions and publications using structural equation modeling (SEM. Chin (1998) considera que para $\mathrm{R}^{2}$ ser fraco o valor situa-se em torno de 0,19; para ser moderado, o valor está centrado em 0,33 e finalmente para ser substancial, o valor esperado é 0,67.

\section{Análise dos Resultados}

A análise dos dados demográficos indicou predomínio de jovens, entre 20 a 29 anos (62,70\%), solteiros (63,27\%), situados em classe intermediária de renda, de 1,5 mil a 3,5 mil reais (46\%).

As ligações substanciais e os resultados para o teste de hipóteses indicam que há evidência sugestiva contra $\mathrm{HO}$ nas relações Prazer - Funcional $(0,059)$ e Prazer - Exclusividade $(0,137)$ e evidência muito forte contra HO nas relações Prazer - Tradição $(0,000)$ e Prazer - Status $(0,006)$.

Quadro 1: Relação entre Variáveis Latentes

\begin{tabular}{lcccc}
\hline & Caminho & Estatística $\boldsymbol{t}$ & $\mathbf{R}^{2}$ & $\boldsymbol{p}$-value \\
\hline Prazer » & Tradição & 1,406 & 0,215 & 0,000 \\
Prazer » & Status & 2,613 & 0,180 & 0,006 \\
Prazer » & Funcional & 2,463 & 0,137 & 0,059 \\
Prazer » & Exclusividade & 2,765 & 0,111 & 0,137 \\
\hline
\end{tabular}

É perceptível que o construto prazer não explica de forma isolada o consumo masstige e que tradição, exclusividade e diferenciação se apresentam igualmente importantes. O prazer em consumir é formado pela tradição advinda das marcas; do status que envolve esse consumo e dos atributos intrínsecos aos produtos e serviços relacionados. 
Em três pesquisas recentes, as dimensões pesquisadas eram ligeiramente distintas. No estudo francês era previsto: [1] superioridade do produto; [2] distribuição seletiva; [3] classe e status; [4] originalidade; [5] exclusividade; [6] glamour; [7] raridade e [8] fashion (Kapferer \& Valette-Florence, 2016). No estudo norte americano as dimensões foram constituídas por [1] diferenciação, [2] qualidade extrema, [3] hedonismo, [4] singularidade e [5] projeção pessoal (Vigneron \& Johnson, 2017). Em estudo realizado no Reino Unido, as dimensões propostas contemplaram [1] afeto, [2] características, [3] status, [4] presentear alguém (gifting) e [5] envolvimento (Walley, Custance, Copley, \& Perry, 2013). As dimensões envolvidas sugerem uma questão semântica, que suscita outros estudos, podendo inclusive contemplar aspectos como a reputação corporativa (Schwartz, 1992; Walsh, Schaarschmidt, \& Ivens, 2017).

A figura 2 indica a distribuição homogênea para a maioria das variáveis relacionadas, exceto para três variáveis: [...] identificação daqueles que têm dinheiro'; [...] reflete um estilo de estilo de vida e [...] acerca do conhecimento do universo das grandes marcas.

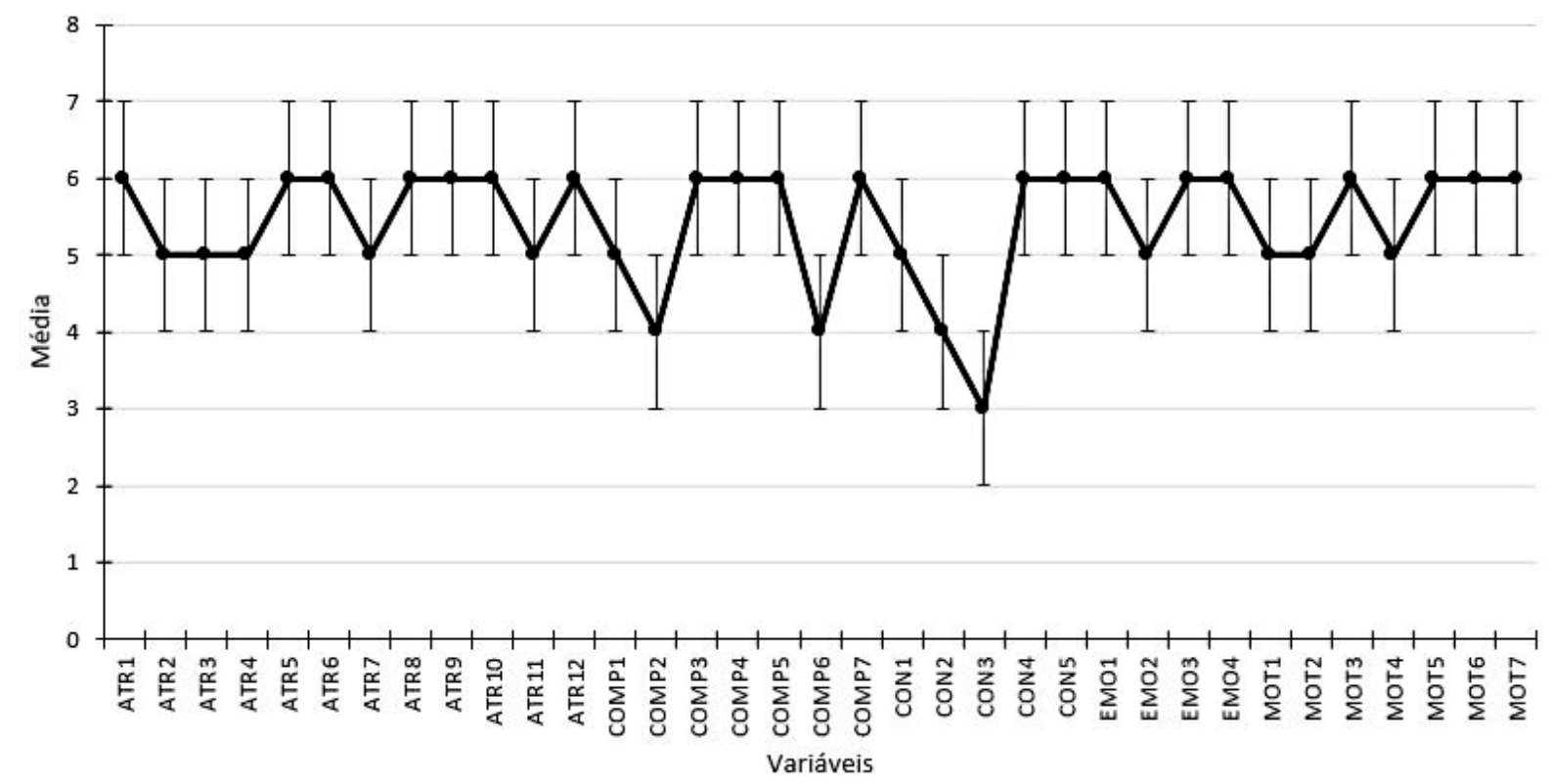

Figura 2. Média e Erro

A dimensão prazer representa um construto importante para o consumo em questão. Alinhado a estudos semelhantes, depreende-se que os consumidores podem "internalizar" o masstige se motivados por prazeres estéticos e sensibilidade cultural ou ainda por questões pessoais de acordo com determinados valores (Amatulli \& Guido, 2011). Esse masstige internalizado pode estar ligado a conceitos como prazer autodirecionado, uma indulgência, bem como congruência com o self interno (Tsai, 2005). Também é possível relacionar que o prazer é elaborado numa relação emocional com o consumo conspicuamente ou não (Petrescu \& Kara, 2018; Makkar \& Yap, 2018).

Para aqueles consumidores motivados puramente pelo prazer, há menos interessados no preço do que na qualidade, nas propriedades do 
produto e no desempenho de consumo. O preço serve apenas como prova de qualidade para esses consumidores (Uzgoren \& Guney, 2012; Vigneron \& Johnson, 2017).

Os valores para $p$-value sugerem forte relação entre prazer e os atributos, tradição, funcionalidade-atributos e exclusividade, entretanto há ressalvas a serem feitas com relação à qualidade psicométrica do modelo. Tais questões apontam para limitações para o uso dos resultados.

1. Estabilidade: coeficiente de correlação intraclasse (intraclass correlation coefficient, ICC) indicou 0,810, indicador acima do parâmetro 0,800, portanto, considerado satisfatório. O teste para estabilidade de uma medida é o grau em que resultados similares são obtidos em dois momentos distintos, ou seja, é a estimativa da consistência das repetições das medidas;

2. Consistência interna: os indicadores para o Alfa de Cronbach variaram de 0,626 a 0,874, sendo considerados satisfatórios no âmbito das Ciências Sociais Aplicadas.

3. Validade do Constructo: A avaliação da Variância Média Extraída (AVE's) revelou-se satisfatória, variando de 0,646 a 0,731 (ver Tabela 3).

4. Qualidade de ajuste do modelo: avaliada pelos coeficientes de determinação de Pearson $\left(R^{2}\right)$, quando valores iguais a $2 \%$ são classificados como efeito pequeno, 13\% como efeito médio e $26 \%$ como efeito grande. As relações entre os construtos variaram de $11 \%$ a $21,5 \%$, sendo classificados como de efeito médio.

Outros testes são sugeridos para a avaliação psicométrica do modelo, nesse caso, os mais importantes foram realizados. Sugere-se consulta em Souza e Guirardello (2017).

Os 4 pontos acima relacionados evidenciam a busca pela confiabilidade do modelo. Outros pontos criam dificuldades para generalização desse instrumento e dos achados da pesquisa:

[1] Trata-se de um tema abstrato. O conceito masstige empregado é de complexa definição e sofre interferência de fatores demográficos como idade, renda e região;

[2] Algumas culturas tendem a valorizar o masstige, enquanto outras enaltecem a tradição das grandes marcas (Choi, 2011; Reboux 2014);

[3] Agravamento nos cenários econômicos. Crises econômicas tendem a impelir o consumo para opções substitutas e marcas menos prestigiadas.

\section{Discussão e Implicações}

Trabalhou-se nesse artigo a hipótese central de que o prazer é a mola propulsora do consumo posicional e acessível, sendo a materialização do prazer como egoísmo ético. A dimensão 'egoísmo ético - prazer' foi analisada a partir dos construtos 'diferenciação', 'status', 'exclusividade', 
'tradição' e 'funcional'. Vale ressaltar que o construto 'funcional' contempla também os atributos do produto, como preço, garantia e qualidade. Essas são questões chaves para a busca da distinção por autores como Thorstein Veblen e Pierre Bourdieu.

As relações que levam ao prazer apresentaram conexões significativas no sentido de aceitar a maioria das hipóteses relacionadas. A hipótese 1 (a tradição influencia o prazer no consumo posicional e masstige), a hipótese 2 (status influencia o prazer no consumo posicional e masstige) e a hipótese 4 (o funcional influencia o prazer no consumo posicional e masstige) foram aceitas. A hipótese 3 não foi confirmada (a exclusividade influencia o prazer no consumo posicional e masstige). A tradição, o status e as características funcionais do produto revelam-se estruturais nesse consumo.

A tradição (percebida como o artesanato e a história da marca) despontou como o mais importante construto, a despeito de fatores como a economia digital e aceleração do lançamento de novos produtos que fragmentam o apelo das marcas tradicionais (Liu, Perry, \& Gadzinski, 2019).

Os achados do estudo trazem contribuições práticas para o consumo masstige. A tradição, conhecida como o feito à mão, o tradicional e o clássico, renasce para o marketing contemporâneo com nova roupagem. A figura do artesão fundador da grande marca do luxo clássico cedeu espaço para uma série de profissionais nascidos na era da economia digital que informam seus seguidores dos reais significados de cada marca (Tynan, McKechnie, \& Chhuon, 2010).

A tradição também não é vista mais como um artesanato. Não se trata de criar um produto ou serviço de forma manual, mas informar que alguma parte do processo é feita à mão, tal como ajustes em peças de roupa (Wang \& Cho, 2012). Tais pontos explicam, em parte, por que o produto usado (second hand) de uma marca forte pode ser mais valorizado do que um produto novo, oriundo de uma marca desconhecida (Zampier, Rita de Cássia \& Pinto, 2019).

Os testes realizados com as hipóteses na dimensão egoísmo ético- prazer confirmaram questões importantes nessa modalidade de consumo: a exclusividade não é estrutural no consumo masstige. Quando se adquire produtos de marcas masstige, ignora-se o alto preço que sustenta a exclusividade e efetivamente busca-se baixos preços aliados à diversidade. É o que tem ocorrido com as marcas Uniqlo (Choi, 2011), Ralph Laurent e Calvin Klein (Truong, McColl, \& Kitchen, 2009).

\section{Referências}

Allérès, D. (2000). Luxe... : Stratégies, Marketing. (4ª ed.). Paris: Economica.

Amatulli, C., \& Guido, G. (2011). Determinants of purchasing intention for fashion luxury goods in the Italian market A laddering approach. Journal of Fashion Marketing and Management: An International Journal, 15(1), 123-136.

Beverland, M. (2006). The 'real thing': branding authenticity in the luxury wine trade. Journal of Business Research, 59(2), 251-258. 
Baudrillard, J. (1995). A sociedade de consumo. Rio de Janeiro: Elfos.

Bourdieu, P. (1989). O poder simbólico. Rio de Janeiro: Bertrand Brasil.

Bourdieu, P. (2005). A economia das trocas simbólicas (6a ed). Rio de Janeiro: Perspectiva.

Chin, W. W. (1998). Commentary: Issues and Opinion on Structural Equation Modeling. MIS Quarterly, 22(1), 1.

Choi, E. (2011). The rise of Uniqlo: leading paradigm change in fashion business and distribution in Japan. Entreprises et Histoire, 64(3), 85. https://doi.org/10.3917/ eh.064.0085

Dawkins, R. (1993). El gen egoísta Las bases biológicas de nuestra conducta. Barcelona: Oxford University Press.

Douglas, M., \& Isherwood, B. (2006). The world of goods. London: Routlege.

Dubois, B., Czellar, S., \& Laurent, G. (2005). Consumer Segmentation Based on Attitudes Toward Luxury: Empirical Evidence from Twenty Countries. Marketing Letters, 16(2), 115-128.

Fiske, N., \& Silverstein, M. (2004). Trading Up: Trends, Brands, and Practices 2004 Research Update. Retrieved July 11, 2006, from http://www.bcg.com/ publications/ publications_search_results.jsp?PUBID=1120S.

Giannetti, E. (1994). A Fábula das Abelhas. Braudel Papers - Fundação Armando Alvares Penteado, (5), 3-15.

Hair, J. F., Black, W. C., Babin, B. J., Anderson, R. E., \& Tatham, R. L. (1998). Multivariate Data Analysis (5th ed.). Upper Saddle River: Prentice Hall.

James, W., Burkhardt, F., Bowers, F., \& Skrupskelis, I. K. (2017). The Principles of Psychology. London: Createspace Independent Publishing Platform.

Kapferer, J. N., \& Valette-Florence, P. (2016). Beyond rarity: the paths of luxury desire. How luxury brands grow yet remain desirable. Journal of Product \& Brand Management, 25, 120-133.

Liu, S., Perry, P., \& Gadzinski, G. (2019). The implications of digital marketing on WeChat for luxury fashion brands in China. Journal of Brand Management, 26(4), 395-409.

Lipovetsky, G., \& Roux, E. (2005). O Luxo Eterno. São Paulo: Companhia das Letras.

Makkar, M., \& Yap, S. (2018, February). Journal of Retailing and Consumer Services Emotional experiences behind the pursuit of inconspicuous luxury. Journal of Retailing and Consumer Services, 44, 222-234. https://doi.org/10.1016/j. jretconser.2018.07.001

Mandeville, B. (2017). A fábula das abelhas: ou vícios privados, benefícios públicos (2nd ed.). São Paulo: Fundação Editora da Unesp.

McCracken, G. (1986). Culture and Consumption: A Theoretical Account of the Structure and Movement of the Cultural Meaning of Consumer Goods. Journal of Consumer Research, 13(1), 71-84. https://doi.org/http://dx.doi.org/10.1086/209048

Paul, J. (2019). Masstige model and measure for brand management. European Management Journal, 37(3), 299-312. https://doi.org/10.1016/j.emj.2018.07.003

Petrescu, M., \& Kara, A. (2018). Consumer Aspirations and Subjective WellBeing. Journal of International Consumer Marketing, 30(5), 304-316 
Pinker, S. (2009). Como a mente funciona. São Paulo: Companhia das Letras.

Reboux, B. (2014). UNIQLO: A stitch in time. In P. Haghirian (Ed.). Case studies in Asian management (Chap. 2, pp. 87-102). Singapore: World Scientific.

Serraf, G. (1991). Le produit de luxe: somptuaire ou ostentatoire? Revue Francaise de Marketing, 2(3), 7-15.

Silverstein, M., \& Fiske, J. (2003). N. Luxury for the masses. Harvard business review, 81(4), 48-59.

Smith, A. (2017). A riqueza das nações. São Paulo, Nova Fronteira.

Solomon, M., Russel-Bennett, R., \& Previte, J. (2002). Consumer behaviour. Pearson Higher Education.

Souza, A. C. D., Alexandre, N. M. C., \& Guirardello, E. D. B. (2017). Psychometric properties in instruments evaluation of reliability and validity. Epidemiologia e Serviços de Saúde, 26, 649-659.

Truong, Y., McColl, R., \& Kitchen, P. J. (2009). New luxury brand positioning and the emergence of. Journal of Brand Management, 16(5), 375-382. https://doi. org/10.1057/bm.2009.1

Tsai, S. (2005). Impact of personal orientation on luxury- brand purchase value : an international investigation. International Journal of Market Research, 47, 177-206.

Tynan, C., McKechnie, S., \& Chhuon, C. (2010). Co-creating value for luxury brands. Journal of Business Research, 63(11), 1156-1163. https://doi.org/10.1016/j. jbusres.2009.10.012

Uzgoren, E., \& Guney, T. (2012). The snop effect in the consumption of luxury goods. Procedia - Social and Behavioral Sciences, 62(1), 628-637. https://doi.org/10.1016/j. sbspro.2012.09.105

Valette-Florence, P., Kapferer, J. N., \& Valette-Florence, P. (2016). Beyond rarity: the paths of luxury desire. How luxury brands grow yet remain desirable. Journal of Product \& Brand Management, 25(2), 120-133. https://doi.org/10.1108/JPBM-092015-0988

Veblen, T. (2017). The Theory of the Leisure lass. London: Routledge.

Vigneron, F., \& Johnson, L. W. (2017). Measuring perceptions of brand luxury. Journal of Brand Management, 11, 484-506. https://doi.org/10.1007/978-3-319-51127-6

Zampier, R. L., Rita de Cássia, P. F., \& Pinto, M. R. (2019). Authenticity in discursive practices of the online market for second-hand luxury clothing. The Qualitative Report, 24(12), 3125-3149.

Walley, K., Custance, P., Copley, P., \& Perry, S. (2013). The key dimensions of luxury from a UK consumers' perspective. Marketing Intelligence \& Planning, 31(7), 823-837. https://doi.org/10.1108/MIP-09-2012-0092

Walsh, G., Schaarschmidt, M., \& Ivens, S. (2017). Effects of customer-based corporate reputation on perceived risk and relational outcomes: empirical evidence from gender moderation in fashion retailing. Journal of Product \& Brand Management, 26(3), 227-238. https://doi.org/10.1108/JPBM-07-2016-1267

Wang, Y., \& Cho, H. (2012). The effect of fashion innovativeness on consumer's online apparel customization. International Journal of Organizational Innovation, 5(2), 263-283 